\title{
THE HEAT FLOW AND HARMONIC MAPS ON A CLASS OF MANIFOLDS
}

\begin{abstract}
XiAO ZHANG
We study the heat flow for harmonic maps from a complete noncompact manifold $M$ which satisfies conditions (a) and (b) in $\S 1$. We show that if the target manifold $N$ is complete, the $C^{2}$ initial map has bounded image in $N$ and has bounded energy density and bounded tension field, then the short-time solution of (1.1) in $\S 1$ exists and is unique. Additional, if the sectional curvature of $N$ is bounded from above, either the long-time solution of (1.1) exists or the energy density of heat flow blows up at a finite time. Moreover, if $N$ has nonpositive sectional curvature and (1.1) has a long-time solution $u(\cdot, t)$ whose energy density increases logarithmically, and there is a point $p \in M$ and a sequence $t_{\nu} \rightarrow \infty$ such that $u\left(\cdot, t_{\nu}\right)$ converges uniformly on compact subsets of $M$ to a harmonic map $u_{\infty}$ by passing to a subsequence.

For this class of manifolds which satisfy (a) and (b), we also get $L^{p}(p>0)$ mean-value inequalities for subsolutions of heat equations and gradient estimates for solutions of heat equations.
\end{abstract}

\section{Introduction.}

Let $M^{m}$ and $N^{n}$ be two complete Riemannian manifolds with their metrics given locally by $d s_{M}^{2}=g_{i j} d x^{i} d x^{j}$ and $d s_{N}^{2}=h_{\alpha \beta} d u^{\alpha} d u^{\beta}$ respectively. For any differentiable map $u$ from $M$ to $N$, the energy density of $u$ at $x \in M$ is defined by

$$
e(u)(x)=g^{i j} \frac{\partial u^{\alpha}}{\partial x^{i}} \frac{\partial u^{\beta}}{\partial x^{j}} h_{\alpha \beta}(u)(x),
$$

where $\left(g^{i j}\right)=\left(g_{i j}\right)^{-1}$. The total energy of $u$ is given by

$$
E(u)(x)=\int_{M} e(u)(x) d x
$$

The map $u$ is called a harmonic map if it is a classical solution of the EularLagrange equation of the total energy functional, which can be written as

$$
\tau(u)(x)=0
$$




$$
\tau^{\alpha}(u)(x)=\triangle u^{\alpha}(x)+g^{i j} \frac{\partial u^{\beta}}{\partial x^{i}} \frac{\partial u^{\gamma}}{\partial x^{j}} \Gamma_{\beta \gamma}^{\alpha}(u)(x),
$$

for $\alpha=1, \cdots, n$, where $\triangle$ is the Laplace-Beltrami operator on $M, \Gamma_{\beta \gamma}^{\alpha}$ are the Christoffel symbols on $N . \tau(u)$ is called the tension field of $u$. The corresponding parabolic system defined on $M \times[0, \infty)$ with initial data $h$ is given by

$$
\frac{\partial u}{\partial t}=\tau(u), \quad u(x, 0)=h(x)
$$

which is called the heat equation for harmonic maps and solution $u$ is called a heat flow.

When $M$ and $N$ are compact Riemannian manifolds without boundary and $N$ has nonpositive sectional curvature, Eells and Sampson [E-S] proved that any $C^{1}$ map from $M$ into $N$ can be deformed to a harmonic map by solving (1.1). The analogous version for compact manifolds with boundary was proved by Hamilton [H]. If $M$ is complete noncompact and $N$ is compact with nonpositive sectional curvature, Schoen and Yau [S-Y1] proved that any $C^{1}$ map from $M$ into $N$ with finite total energy can be deformed on any subsets of $M$ to a harmonic map with finite total energy. Their method based on the Hamilton's results. Later Liao and Tam [Lo-T] recovered their results by studying the heat flow directly. Li and Tam $[\mathbf{L}-\mathbf{T}]$ considered the case when both $M$ and $N$ are complete noncompact Riemannian manifolds and developed general method to study harmonic maps on noncompact manifold via heat flow. One of their main results is: Let $M$, $N$ be complete noncompact Riemannian manifolds, $\operatorname{Ricci}^{M} \geq-K(K>0)$. Let $h \in C^{1}(M, N)$ with bounded energy density such that $h(M)$ is also bounded. Then there exists $T_{0}>0$ and a unique map $u$ which satisfies (1.1) on $M \times\left[0, T_{0}\right)$. If, in addition, $\operatorname{Riem}^{N} \leq 0$, then (1.1) has a unique solution $u$ on $M \times[0, \infty)$ which satisfies that for all $T>0, u(M \times[0, T])$ is bounded, and $\sup _{M \times[0, T]} e(u)<\infty$. Furthermore, if there exists a point $p \in M$ and a sequence $t_{v} \rightarrow \infty$ such that $u\left(p, t_{v}\right)$ converges in $N$, then by passing to a subsequence, $u\left(\cdot, t_{v}\right)$ converges uniformly on compact subsets together with their first and second derivatives to a harmonic map $u_{\infty}$.

The Bochner formula plays a role for proving the above theorems, but it depends on the lower bound of the Ricci curvature on the domain manifold extremely. Thus, when the domain manifold is only assumed to be complete noncompact without boundary and satisfy the following two conditions:

(a) There exists a constant $A>1$ such that for any $x \in M$ and for all $R>0$

$$
V_{x}(2 R) \leq A V_{x}(R)
$$


(b) There exist constants $N>1, a>0$ such that for any function $f \in$ $C^{\infty}\left(B_{x}(N R)\right)$

$$
\frac{a}{R^{2}} \inf _{\alpha \in R} \int_{B_{x}(R)}(f-\alpha)^{2} \leq \int_{B_{x}(N R)}|\nabla f|^{2}
$$

we will lose many importment estimates obtained via the Bochner formula. This class of manifolds were introduced and studied by Grigor'yan [G]. Obviously, (a), (b) are quasi-isometric invariant (with possibly different $A, a$ and $N$ ). It is known that if $M$ has nonnegative Ricci curvature, then $M$ satisfies (a), (b), see [G]. Hence this class of manifolds includes noncompact manifolds which are quasi-isometric to manifolds with nonnegative Ricci curvature. By using the distance function of target manifold and the well-known fact that the composition of a convex function in the target with a harmonic map is a subharmonic function of the domain, Tam [T1], [T2] has got some results which assert that any harmonic map that has a bounded image in target manifold or has a bounded total energy from this class of manifolds to simply connected, nonpositive sectional curvature manifolds must be constant map. These generalized the theorems of Cheng $[\mathbf{C}]$ and Schoen-Yau [S-Y1]. In this paper, we will prove the following theorem:

Main Theorem. Let $M$ be a complete noncompact manifold without boundary and satisfy the conditions (a) and (b), $N$ be an arbitrary complete manifold.

(i) Given $h \in C^{2}(M, N)$ such that $h(M)$ is bounded in $N$, the energy density $e(h)$ and the tension field $\tau(h)$ are also bounded on $M$. Then there exists $T_{0}>0$ such that (1.1) has a unique solution $u(x, t)$ on $M \times\left[0, T_{0}\right)$. If, in addition, $\operatorname{Riem}^{N} \leq k(k \geq 0)$, let $T^{*}$ be the supremum of those $T$ such that (1.1) has a unique solution $u(x, t)$ on $M \times[0, T)$ and $\sup _{M \times[0, T)} e(u)<\infty$, then eithor $T^{*}=\infty$ or $T^{*}<\infty$ and $\lim _{T \rightarrow T^{*}} \sup _{M \times[0, T)} e(u)=\infty$.

(ii) Suppose $\mathrm{Riem}^{N} \leq 0$ and (1.1) has a long-time solution $u(x, t)$ on $M \times[0, \infty)$. If $s(t)=\sup _{M \times[0, t)} e(u)(x, t)=O(\log t)$, and there exists a point $p \in M$ and a sequence $t_{\nu} \rightarrow \infty$ such that sequence $u\left(p, t_{\nu}\right)$ converges in $N$, then $u\left(\cdot, t_{\nu}\right)$ converges uniformly on compact subsets of $M$ to a harmonic map $u_{\infty}$ by passing to a subsequence.

Here, we need some stronger assumptions on the initial data $\left(C^{2}\right.$, bounded energy density and tension field) in order to get some key estimates without using the Bochner formula (Theorem 3.4(iv), etc.). Perhaps, good estimates on the heat kernel for 1-form on this class of manifolds might weaken our assumptions on the initial data.

This paper is arranged as follows: 
In the second section, we will give some known results which were proved by Grigor'yan in [G], and generalize a mean-value inequality of Grigor'yan's about the subsolution of the heat equation on this class of manifolds.

In the third section, we will derive estimates for solutions of the homogeneous and inhomogenous heat equations on these manifolds.

In the fourth and fifth sections, we will consider the questions of both the short-time and the long-time existences of solutions for (1.1). And the convergence to a harmonic map as time tends to infinity. We will prove the Main Theorem in these two sections.

Acknowledgements. This work was done while the author was a graduate student in the Department of Mathematics, The Chinese University of Hong Kong. The author would like to thank Prof. S.Y. Cheng and Prof. S.T. Yau for their encouragement, Prof. L.F. Tam for suggesting this problem and his helpful discussions, and Dr. Tom Y.H. Wan for his interest in this work and valuable discussions.

The author would also like to thank Prof. W.Y. Ding and Prof. H.D. Chao for pointing out some errors in the early version.

\section{Mean-value inequalities.}

In this section, we will first give some known theorems about the volume comparison for balls, the Harnack inequality for the positive solution of heat equation, and the heat kernel estimates which were proven in $[\mathbf{G}]$ on a class of complete noncompact Riemannian manifolds satisfying (a) and (b), we will also prove some mean-value inequalites for the subsolution of the heat equation on these manifolds and generalize the result of Grigor'yan's (see [G], Theorem 3.1).

Theorem 2.1. Let $M$ be a complete noncompact manifold which satisfies (a) and (b), then

(i) (Volume comparison). For all $x \in M$ and all $R \geq r>0$,

$$
\left(1+A^{3}\right)^{-1}\left(\frac{R}{r}\right)^{\alpha} \leq \frac{V_{x}(R)}{V_{x}(r)} \leq A^{3}\left(\frac{R}{r}\right)^{\beta}
$$

where $\alpha=\log _{3}\left(1+A^{-3}\right), \beta=\log _{2} A$.

(ii) (Harnack inequality). For any positive solution $u(x, t)$ of heat equation, on $M \times[0, \infty)$, for any $x, y$ in $M$ and $T>t>0$,

$$
u(x, t) \leq u(y, T) \exp \left(C_{1}\left(\frac{T}{t}+\frac{r(x, y)^{2}}{T-t}\right)\right),
$$

where $r(x, y)$ is the distance function between $x$ and $y$, and $C_{1}$ is a constant depending only on $A$, a, and $N$. 
(iii) (Heat kernel estimates). Let $H(x, y, t)=\lim _{R \rightarrow \infty} H_{R}(x, y, t)$ be the fundamental solution of the heat equation obtained by compact exhaustion, where $H_{R}(x, y, t)$ is the heat kernel of $B_{p}(R)$ with Dirichlet boundary value, $p$ is a fixed point in $M$. Then

$$
\frac{C_{2}}{V_{x}(\sqrt{t})} \exp \left(-\frac{C_{3} r(x, y)^{2}}{t}\right) \leq H(x, y, t) \leq \frac{1}{C_{2} V_{x}(\sqrt{t})} \exp \left(-\frac{C_{4} r(x, y)^{2}}{t}\right),
$$

where $C_{4}<\frac{1}{4}$ is a constant, $C_{3}>0$ is a constant depending only on $A, a$, and $N, C_{2}>0$ is a constant depending only on $A, a, N$ and $C_{4}$.

Proof. See $[\mathrm{G}]$.

We have known (see [G], Theorem 1.4) that if complete noncompact manifold $M$ satisfies (a) and (b), then in each ball $B_{x}(R)$ there is an isoperimetric inequality

$$
\lambda_{1}(\Omega) \geq \Lambda(\operatorname{Vol}(\Omega))
$$

with function

$$
\Lambda(v)=\frac{b}{R^{2}}\left(\frac{V_{x}(R)}{v}\right)^{2 / \beta},
$$

where $\Omega$ is the domain in $M, \lambda_{1}(\Omega)$ is the first eigenvalue of $\Omega$ and $b>0$ is a constant depending only on $A, a$ and $N$.

The function $\frac{v}{\Lambda(v)}$ is obviously strictly monotonically increasing on $(0, \infty)$ with range $(0, \infty)$. It therefore has an inverse function on $(0, \infty)$, which we denote by $\omega$. We define functions $V(t)$ and $W(r)(t>0, r>0)$ by the equations

$$
C_{5} t=\int_{0}^{V(t)} \frac{d \xi}{\omega(\xi)}, \quad C_{6} r=\int_{0}^{W(r)} \frac{d \xi}{\sqrt{\xi \omega(\xi)}}
$$

where $C_{5}, C_{6}>0$ are constants which will be determined in the proof. Everywhere below we assume that the integrals in (2.2) converge to zero as $t, r \rightarrow 0$.

It is easy to derive from (2.1) that

$$
V(t)=C_{7} \frac{V_{x}(R)}{R^{\beta}} t^{1+\beta / 2}, \quad W(r)=C_{8} \frac{V_{x}(R)}{R^{\beta}} r^{2+\beta},
$$

where $\beta=\log _{2} A, C_{7}, C_{8}>0$ are constants depending only on $C_{5}, C_{6}, A, a$ and $N$. 
Theorem 2.2. $\quad$ Let $M$ be a complete noncompact Riemannian manifold without boundary and satisfy (a) and (b). Suppose the function $u \in$ $C^{\infty}\left(B_{x}(R) \times[0, T]\right)$ satisfies

$$
\left(\triangle-\frac{\partial}{\partial t}\right) u \geq 0
$$

(i) For any $0<\delta<1,0<\tau \leq T$ and $p>0$, there exists a constant $C>0$ depending only on $p, A, a$, and $N$ such that

$$
\sup _{B_{x}((1-\delta) R) \times[\tau, T]} u_{+}^{p} \leq \frac{C}{\sigma^{1+\beta / 2}} \frac{R^{\beta}}{V_{x}(R)} \int_{0}^{T} \int_{B_{x}(R)} u_{+}^{p} .
$$

Where $\sigma=\min \left(\tau, \delta^{2} R^{2}\right), \beta=\log _{2} A$, and $u_{+}$is the positive part of $u$.

(ii) Let $\bar{u}=u-\sup _{B_{x}(R)}|u(y, 0)|$, then there exists $C>0$ depending only on $p, A, a$ and $N$ such that

$$
\sup _{B_{x}((1-\delta) R) \times[0, T]} \bar{u}_{+}^{p} \leq \frac{C}{\delta^{2+\beta} R^{2} V_{x}(R)} \int_{0}^{T} \int_{B_{x}(R)} \bar{u}_{+}^{p} .
$$

In particular, if $p \geq 1$, then

$$
\sup _{B_{x}((1-\delta) R) \times[0, T]} u_{+}^{p} \leq \frac{2^{p-1} C}{\delta^{2+\beta} R^{2} V_{x}(R)} \int_{0}^{T} \int_{B_{x}(R)} u_{+}^{p}+2^{p-1} \sup _{B_{x}(R)}|u(y, 0)|^{p} .
$$

Before proving it, we will prove some lemmas.

Lemma 2.3. Suppose, under the conditions of Theorem 2.2, that $v=$ $(u-\theta)_{+}$, where $\theta \geq 0$ is an arbitrary number. Let $\eta(y, t)$ be a Lipschitz function in $M \times[0, \infty)$ such that $\operatorname{supp}(\eta(y, t)) \subset \subset \overline{B_{x}}(R)$ for $t \geq 0$. Then for $p>1$, there exists $C>0$ depending only on $p$ such that

$$
\begin{aligned}
& \int_{B_{x}(R)}\left(v^{p} \eta^{2}\right)(y, t)+\frac{p-1}{p} \int_{0}^{t} \int_{B_{x}(R)}\left|\nabla\left(v^{\frac{p}{2}} \eta\right)\right|^{2} \\
& \leq C \int_{0}^{t} \int_{B_{x}(R)} v^{p}\left(|\nabla \eta|^{2}+\eta \eta_{t}\right)+\int_{B_{x}(R)}\left(v^{p} \eta^{2}\right)(y, 0) .
\end{aligned}
$$

Proof.

$$
\int_{B_{x}(R)} v^{p-1} v_{t} \eta^{2} \leq \int_{\{u>\theta\}} v^{p-1} \triangle u \eta^{2}
$$




$$
\begin{aligned}
& =\int_{\{u=\theta\}} \frac{\partial u}{\partial \nu} v^{p-1} \eta^{2}-\int_{\{u>\theta\}} \nabla u \nabla\left(v^{p-1} \eta^{2}\right) \\
& =-\int_{B_{x}(R)} \nabla v \nabla\left(v^{p-1} \eta^{2}\right) .
\end{aligned}
$$

Integrating the above inequality from 0 to $t$, we get

$$
\begin{aligned}
\int_{0}^{t} \int_{B_{x}(R)} v^{p-1} v_{t} \eta^{2} \leq & -(p-1) \int_{0}^{t} \int_{B_{x}(R)} v^{p-2} \eta^{2}|\nabla v|^{2} \\
& -2 \int_{0}^{t} \int_{B_{x}(R)} v^{p-1} \eta \nabla v \nabla \eta
\end{aligned}
$$

Since

$$
v^{p-1} \eta \nabla v \nabla \eta \leq \frac{p}{4} v^{p-2} \eta^{2}|\nabla v|^{2}+\frac{1}{p} v^{p}|\nabla \eta|^{2},
$$

then

$$
\left|\nabla\left(v^{\frac{p}{2}} \eta\right)\right|^{2} \leq \frac{p^{2}}{2} v^{p-2} \eta^{2}|\nabla v|^{2}+2 v^{p}|\nabla \eta|^{2}
$$

therefore

$$
v^{p-2} \eta^{2}|\nabla v|^{2} \geq \frac{2}{p^{2}}\left|\nabla\left(v^{\frac{p^{2}}{2}} \eta\right)\right|^{2}-\frac{4}{p^{2}} v^{p}|\nabla \eta|^{2} .
$$

On the other hand,

$$
-2 v^{p-1} \eta \nabla u \nabla \eta \leq \frac{p-1}{2} v^{p-2} \eta^{2}|\nabla v|^{2}+\frac{2}{p-1} v^{p}|\nabla \eta|^{2} .
$$

Substituting (2.10), (2.11) into (2.9),

$$
\begin{aligned}
& \int_{0}^{t} \int_{B_{x}(R)} v^{p-1} v_{t} \eta^{2} \\
& \leq-\frac{p-1}{p} \int_{0}^{t} \int_{B_{x}(R)}\left|\nabla\left(v^{\frac{p}{2}} \eta\right)\right|^{2}+\left(\frac{4}{p^{2}}+\frac{2}{p-1}\right) \int_{0}^{t} \int_{B_{x}(R)} v^{p}|\nabla \eta|^{2} .
\end{aligned}
$$

Since

$$
\int_{0}^{t} \int_{B_{x}(R)} v^{p-1} v_{t} \eta^{2}=\frac{1}{p} \int_{B_{x}(R)}\left(\left(v^{p} \eta^{2}\right)(y, t)-\left(v^{p} \eta^{2}\right)(y, 0)\right)-\frac{2}{p} \int_{0}^{t} \int_{B_{x}(R)} v^{p} \eta \eta_{t} .
$$

Hence we get (2.8), here, $C=\frac{4}{p}+\frac{2 p}{p-1}$. 
Lemma 2.4. Suppose, under the conditions of Theorem 2.2, that $p>1$, for any $\theta>0$, let

$$
H=\int_{0}^{T} \int_{B_{x}(R)} u_{+}^{p}, \quad \bar{H}=\int_{\tau}^{T} \int_{B_{x}((1-\delta) R)}(u-\theta)_{+}^{p},
$$

then there exists $C>0$ depending only on $p$ such that

$$
\bar{H} \leq \frac{C H}{\sigma \Lambda\left(C \sigma^{-1} \theta^{-p} H\right)}
$$

Proof. In (2.8) we set

$$
\eta(y, t)=\eta_{1}(y) \eta_{2}(t)
$$

where $\eta_{1}$ is 1 inside $B_{x}\left(\left(1-\frac{\delta}{2}\right) R\right)$, zero outside $B_{x}(R)$ and linear between $B_{x}\left(\left(1-\frac{\delta}{2}\right) R\right)$ and $B_{x}(R)$; and $\eta_{2}$ is 1 when $t \geq \tau$, zero when $t=0$ and linear between 0 and $\tau$. We also set $v=u_{+}$. For any $t \in[\tau, T]$, since

$$
|\nabla \eta|^{2} \leq \frac{4}{\sigma}, \quad\left|\eta \eta_{t}\right| \leq \frac{1}{\sigma}
$$

then

$$
\int_{B_{x}\left(\left(1-\frac{\delta}{2}\right) R\right)} u_{+}^{p}(y, t) \leq C \int_{0}^{t} \int_{B_{x}(R)} u_{+}^{p}\left(|\nabla \eta|^{2}+\eta \eta_{t}\right) \leq \frac{5 C H}{\sigma}
$$

By setting $\eta_{1}$ is 1 inside $B_{x}((1-\delta) R)$, zero outside $B_{x}\left(\left(1-\frac{\delta}{2}\right) R\right)$ and linear between $B_{x}((1-\delta) R)$ and $B_{x}\left(\left(1-\frac{\delta}{2}\right) R\right)$; and $\eta_{2}$ as before, we have

$$
\int_{0}^{T} \int_{B_{x}(R)}\left|\nabla\left((u-\theta)_{+}^{\frac{p}{2}} \eta\right)\right|^{2} \leq \frac{5 p C}{(p-1) \sigma} \int_{0}^{T} \int_{B_{x}(R)}(u-\theta)_{+}^{p} .
$$

Since for each $t \in[0, T]$,

$$
\operatorname{supp}\left((u-\theta)_{+}^{\frac{p}{2}} \eta\right) \subset \bar{D}_{t},
$$

where

$$
D_{t}=\left\{y \in B_{x}\left(\left(1-\frac{\delta}{2}\right) R\right): u(y, t)>\theta\right\}
$$

then

$$
\int_{B_{x}(R)}\left|\nabla\left((u-\theta)_{+}^{\frac{p}{2}} \eta\right)\right|^{2} \geq \lambda_{1}\left(D_{t}\right) \int_{B_{x}(R)}(u-\theta)_{+}^{p} \eta^{2}
$$

For $t \in[\tau, T]$, it follows from (2.13) that

$$
\operatorname{mes}\left(D_{t}\right) \leq \theta^{-p} \int_{B_{x}\left(\left(1-\frac{\delta}{2}\right) R\right)} u_{+}^{p} \leq 5 C \sigma^{-1} \theta^{-p} H .
$$


By (2.1), (2.14), (2.15) and (2.16), we have

$$
\frac{5 p C}{p-1} \sigma^{-1} H \geq \Lambda\left(5 C \sigma^{-1} \theta^{-p} H\right) \bar{H} \geq \Lambda\left(\frac{5 p C}{p-1} \sigma^{-1} \theta^{-p} H\right) \bar{H} .
$$

This is (2.12).

Lemma 2.5. Suppose, under the conditions of Theorem 2.2, that $p>1$, for any $\theta>0$, let

$$
H^{*}=\int_{0}^{T} \int_{B_{x}(R)} \bar{u}_{+}^{p}, \quad \overline{H^{*}}=\int_{0}^{T} \int_{B_{x}((1-\delta) R)}(\bar{u}-\theta)_{+}^{p},
$$

then there exists $C>0$ depending only on $p$ such that, for $\rho=\delta^{2} R^{2}$.

$$
\overline{H^{*}} \leq \frac{C H^{*}}{\rho \Lambda\left(C \rho^{-1} \theta^{-p} H^{*}\right)} .
$$

Proof. Clearly, $\bar{u}(y, t)$ is also a subsolution of the heat equation and $\bar{u}_{+}(y, 0)=$ 0 on $B_{x}(R)$, by setting $\eta_{2}(t) \equiv 1$ as $t \geq 0$ in the proof of Lemma 2.4, we can get, in a similar way, that

$$
\frac{4 p C}{p-1} \rho^{-1} H^{*} \geq \Lambda\left(4 C \rho^{-1} \theta^{-p} H^{*}\right) \bar{H}^{*} \geq \Lambda\left(\frac{4 p C}{p-1} \rho^{-1} \theta^{-p} H^{*}\right) \bar{H}^{*} .
$$

Thus, the lemma is proved.

Proof of the Theorem 2.2.

Case (i). $\quad p>1$.

For $k=0,1,2, \ldots$, set

$$
t_{0}=0<t_{1}<t_{2}<\ldots \leq \tau, \quad R=r_{0}>r_{1}>r_{2}>\ldots \geq(1-\delta) R,
$$

moreover,

$$
\left(r_{k}-r_{k+1}\right)^{2}=t_{k+1}-t_{k} \equiv \sigma_{k}
$$

Let

$$
\theta^{p}=\frac{H}{\min (V(\tau), W(\delta R))}
$$

set

$$
\theta_{k}=\left(2-2^{-k}\right) \theta
$$


and

$$
H_{k}=\int_{t_{k}}^{T} \int_{B_{x}\left(r_{k}\right)}\left(u-\theta_{k}\right)_{+}^{p} .
$$

Obviously, $H_{k}$ decreases monotonically, and by Lemma 2.4,

$$
H_{k+1} \leq \frac{C H_{k}}{\sigma_{k} \Lambda\left(C \sigma_{k}^{-1} 2^{p(k+1)} \theta^{-p} H_{k}\right)} .
$$

Choose $C_{5}=\left(4^{p} C\right)^{-1}, C_{6}=\left(2^{p} \sqrt{C}\right)^{-1}$ in (2.2), by (2.19),

$$
\int_{0}^{\theta^{-p} H} \frac{d \xi}{\omega(\xi)} \leq \frac{\tau}{4^{p} C}, \quad \int_{0}^{\theta^{-p} H} \frac{d \xi}{\sqrt{\xi \omega(\xi)}} \leq \frac{\delta R}{2^{p} \sqrt{C}} .
$$

Let

$$
\sigma_{k}=\frac{4^{p} C\left(2^{p(-k-1)} \theta^{-p} H\right)}{\omega\left(2^{p(-k-1)} \theta^{-p} H\right)}
$$

for $k \geq 0$, since

$$
\begin{aligned}
0 & \leq t_{k}=\sum_{i=0}^{k} \sigma_{i} \leq \sum_{i=1}^{m+1} \frac{4^{p} C\left(2^{p(-i)} \theta^{-p} H\right)}{\omega\left(2^{p(-i)} \theta^{-p} H\right)} \\
& \leq 4^{p} C \int_{0}^{\infty} \frac{2^{p(-z)} \theta^{-p} H}{\omega\left(2^{p(-z)} \theta^{-p} H\right)} d z=4^{p} C \int_{0}^{\theta^{-p} H} \frac{d \xi}{\omega(\xi)} \leq \tau,
\end{aligned}
$$

and

$$
\begin{aligned}
0 & \leq r_{0}-r_{k}=\sum_{i=0}^{k} \sqrt{\sigma_{i}} \leq \sum_{i=1}^{m+1} \frac{2^{p} \sqrt{C} \sqrt{2^{p(-i)} \theta^{-p} H}}{\sqrt{\omega\left(2^{p(-i)} \theta^{-p} H\right)}} \\
& \leq 2^{p} \sqrt{C} \int_{0}^{\infty} \frac{\sqrt{2^{p(-z)} \theta^{-p} H}}{\sqrt{\omega\left(2^{p(-z)} \theta^{-p} H\right)}} d z=2^{p} \sqrt{C} \int_{0}^{\theta^{-p} H} \frac{d \xi}{\sqrt{\xi \omega(\xi)}} \leq \delta R,
\end{aligned}
$$

where $\xi=2^{p(-z)} \theta^{-p} H$. Thus (2.18) is satisfied and $\sigma_{k}$ is a suitable choice.

We will prove that for all $k=0,1,2, \ldots$,

$$
H_{k} \leq 4^{-p k} H \text {. }
$$

For $k=0,(2.23)$ is obviously satisfied. Suppose (2.23) is satisfied for $k \leq m$. By (2.22), we have

$$
C \sigma_{m}^{-1} 2^{p(-m+1)} \theta^{-p} H=\omega\left(2^{p(-m-1)} \theta^{-p} H\right),
$$


then

$$
\frac{C \sigma_{m}^{-1} 2^{p(-m+1)} \theta^{-p} H}{\Lambda\left(C \sigma_{m}^{-1} 2^{p(-m+1)} \theta^{-p} H\right)}=2^{p(-m-1)} \theta^{-p} H
$$

therefore

$$
\frac{C}{\sigma_{m} \Lambda\left(C \sigma_{m}^{-1} 2^{p(-m+1)} \theta^{-p} H\right)}=4^{-p} .
$$

Thus, by (2.21),

$$
H_{m+1} \leq \frac{C H_{m}}{\sigma_{m} \Lambda\left(C \sigma_{m}^{-1} 2^{p(m+1)} \theta^{-p} 4^{-p m} H\right)}=4^{-p} H_{m} \leq 4^{-p(m+1)} H .
$$

By induction, (2.23) is proved.

Let $k \rightarrow \infty$ in $(2.23)$, we get

$$
\int_{\tau}^{T} \int_{B_{x}((1-\delta) R)}(u-2 \theta)_{+}^{p}=0,
$$

so that

$$
\sup _{B_{x}((1-\delta) R) \times[\tau, T]} u_{+}^{p} \leq 2^{p} \theta^{p} .
$$

Substituting (2.3) into (2.19), we can choose $C>0$ depending only on $p, A, a$, and $N$ such that (2.5) is satisfied.

Case (ii). $0<p \leq 1$.

Let

$$
\delta_{k}=\frac{\delta}{2^{k}}, \quad \tau_{k}=\frac{\tau}{3 \cdot 4^{k}}, \quad M(k)=\sup _{B_{x}\left(\left(1-\delta_{k}\right) R\right) \times\left[\tau_{k}, T\right]} u_{+}^{2} .
$$

By Theorem 2.1(i),

$$
V_{x}\left(\left(1-\delta_{k+1}\right) R\right)^{-1} \leq A^{3}\left(1-\delta_{k+1}\right)^{-\beta} V_{x}(R)^{-1},
$$

let $0<\lambda=1-\frac{p}{2}<1$, then (2.5) implies,

$$
\begin{aligned}
M(k) & \leq \frac{C}{\left(4^{-k-1} \sigma\right)^{1+\beta / 2}} \frac{\left(\left(1-\delta_{k+1}\right) R\right)^{\beta}}{V_{x}\left(\left(1-\delta_{k+1}\right) R\right)} M(k+1)^{\lambda} \int_{\tau_{k+1}}^{T} \int_{B_{x}\left(\left(1-\delta_{k+1}\right) R\right)} u_{+}^{p} \\
& \leq \frac{A^{3} C\left(2^{2+\beta}\right)^{k+1}}{\sigma^{1+\beta / 2}} \frac{R^{\beta}}{V_{x}(R)} M(k+1)^{\lambda} \int_{0}^{T} \int_{B_{x}(R)} u_{+}^{p} .
\end{aligned}
$$

Denote

$$
I=\frac{A^{3} C}{\sigma^{1+\beta / 2}} \frac{R^{\beta}}{V_{x}(R)} \int_{0}^{T} \int_{B_{x}(R)} u_{+}^{p}
$$


Iterating the above inequality, we get

$$
M(0) \leq I^{1+\lambda+\lambda^{2}+\cdots}\left(2^{2+\beta}\right)^{1+2 \lambda+3 \lambda^{2}+\cdots}(M(\infty))^{\lambda^{\infty}}=\left(2^{2+\beta}\right)^{4 / p^{2}} I^{2 / p} .
$$

It is easy to derive our result from this inequality.

For the inequality (2.6), Let

$$
\theta^{p}=\frac{H^{*}}{W(\delta R)}, \quad \theta_{k}=\left(2-2^{-k}\right) \theta, \quad \rho_{k}=\left(r_{k}-r_{k+1}\right)^{2}
$$

and

$$
H_{k}^{*}=\int_{0}^{T} \int_{B_{x}\left(r_{k}\right)}\left(\bar{u}-\theta_{k}\right)_{+}^{p} .
$$

By Lemma 2.5 and the Moser's iteration, (2.6) can be proved by the similar argument.

Since $(|a|+|b|)^{p} \leq 2^{p-1}\left(|a|^{p}+|b|^{p}\right)$ for $p \geq 1$ and $\int_{B_{x}(R)} \bar{u}_{+} \leq \int_{B_{x}(R)} u_{+}$, (2.7) follows.

\section{Gradient estimates.}

In this section, we always denote $M$ as a complete noncompact Riemannian manifold without boundary which satisfies (a) and (b); and $\mathrm{C}$ as an arbitrary positive constant. We will derive various estimates for solutions of homogenous and inhomogenous heat equations on $M$.

Lemma 3.1. For the heat kernel $H(x, y, t)$ of $M$, for any $T>0,0<t<T$ and $p, q \in M$, there exists $C>0$ depending only on $A, a$ and $N$ such that

$$
\int_{M}|H(p, y, t)-H(q, y, t)| d y \leq C \frac{r(p, q)}{\sqrt{t}} .
$$

Proof. By the Harnack inequality (Theorem 2.1(ii)), for the fixed $\delta>0$,

$$
\begin{aligned}
I= & \int_{M}|H(q, y,(1+\delta) t)-H(q, y, t)| d y \\
\leq & \int_{M}\left|\exp \left(C_{1}(1+\delta)\right) H(q, y,(1+\delta) t)-H(q, y, t)\right| d y \\
& +\int_{M}\left(\exp \left(C_{1}(1+\delta)\right)-1\right) H(q, y,(1+\delta) t) d y \\
\leq & 2\left(\exp \left(C_{1}(1+\delta)\right)-1\right)
\end{aligned}
$$




$$
\begin{aligned}
I I= & \int_{M}|H(p, y, t)-H(q, y,(1+\delta) t)| d y \\
\leq & \int_{M}\left|H(p, y, t)-\exp \left(C_{1}(1+\delta)+\frac{r^{2}(p, q)}{\delta t}\right) H(q, y,(1+\delta) t)\right| d y \\
& +\int_{M}\left(\exp \left(C_{1}(1+\delta)+\frac{r^{2}(p, q)}{\delta t}\right)-1\right) H(q, y,(1+\delta) t) d y \\
\leq & 2\left(\exp \left(C_{1}(1+\delta)+\frac{r^{2}(p, q)}{\delta t}\right)-1\right) .
\end{aligned}
$$

Let $s=\frac{\sqrt{C}_{1} r(p, q)}{\sqrt{\delta t}} \geq 0$, therefore, there exists $C^{\prime}>0$ depending only on $C_{1}, \delta$ such that

$$
\int_{M}|H(p, y, t)-H(q, y, t)| d y \leq I+I I \leq C^{\prime}\left(\exp \left(s^{2}\right)-1\right) .
$$

If $s \leq 1$, then

$$
\exp \left(s^{2}\right)-1 \leq(e-1) s^{2} \leq(e-1) s
$$

thus

$$
\int_{M}|H(p, y, t)-H(q, y, t)| d y \leq C^{\prime}(e-1) s=C \frac{r(p, q)}{\sqrt{t}} .
$$

If $s>1$, then

$$
\int_{M}|H(p, y, t)-H(q, y, t)| d y \leq 2<2 s=C \frac{r(p, q)}{\sqrt{t}} .
$$

Lemma 3.2. For any $\alpha>0, T>0$ and $0<t<T$, there exists $C>0$ depending only on $\alpha, A, a$ and $N$ such that

$$
\int_{M} H(x, y, t) r^{\alpha}(x, y) d y \leq C t^{\frac{\alpha}{2}} .
$$

Proof. Let $s=\frac{r}{\sqrt{t}}$, by Theorem 2.1(i), (iii),

$$
\begin{aligned}
\int_{M} H(x, y, t) r^{\alpha} d y= & \int_{B_{x}(2 \sqrt{t})} H(x, y, t) r^{\alpha} d y+\int_{M \backslash B_{x}(2 \sqrt{t})} H(x, y, t) r^{\alpha} d y \\
\leq & (2 \sqrt{t})^{\alpha}+\frac{1}{C_{2} V_{x}(2 \sqrt{t})} \int_{2 \sqrt{t}}^{\infty} \exp \left(-\frac{C_{4} r^{2}}{t}\right) r^{\alpha} d V_{x}(r) \\
= & (2 \sqrt{t})^{\alpha}+\frac{1}{C_{2} V_{x}(2 \sqrt{t})}\left(\left.\exp \left(-\frac{C_{4} r^{2}}{t}\right) r^{\alpha} V_{x}(r)\right|_{2 \sqrt{t}} ^{\infty}\right. \\
& -\int_{2 \sqrt{t}}^{\infty}\left(\exp \left(-\frac{C_{4} r^{2}}{t}\right)\left(-\frac{2 C_{4} r}{t}\right) r^{\alpha}\right.
\end{aligned}
$$




$$
\begin{gathered}
\left.\left.+\alpha \exp \left(-\frac{C_{4} r^{2}}{t}\right) r^{\alpha-1}\right) V_{x}(r) d r\right) \\
\leq\left(2^{\alpha}+\frac{2 C_{4}}{C_{2}} \int_{2}^{\infty} \exp \left(-C_{4} s^{2}\right) s^{1+\alpha} \frac{V_{x}(s \sqrt{t})}{V_{x}(t)} d s\right) t^{\frac{\alpha}{2}} \\
\leq\left(2^{\alpha}+\frac{2 C_{4} A^{3}}{C_{2}} \int_{2}^{\infty} \exp \left(-C_{4} s^{2}\right) s^{1+\alpha+\beta} d s\right) t^{\frac{\alpha}{2}}=C t^{\frac{\alpha}{2}}
\end{gathered}
$$

since the integral of the right hand converges.

Theorem 3.3. Let $f$ be a bounded function on $M \times[0, T]$, and

$$
u(x, t)=-\int_{0}^{t} \int_{M} H(x, y, t-\tau) f(y, \tau) d y d \tau,
$$

for any $0<t<T$, we have

(i)

$$
\sup _{M \times[0, t)}|u| \leq\left(\sup _{M \times[0, t)}|f|\right) t .
$$

(ii)

$$
\sup _{M \times[0, t)}|\nabla u| \leq C\left(\sup _{M \times[0, t]}|f|\right) t^{\frac{1}{2}} .
$$

Proof. (i) Obviously.

(ii). By Lemma 3.1,

$$
\begin{aligned}
|u(x, t)-u(z, t)| & \leq \int_{0}^{t} \int_{M}|H(x, y, t-\tau)-H(z, y, t-\tau)||f(y, \tau)| d y d \tau \\
& \leq C \sup _{M \times[0, t)}|f| \int_{0}^{t} \frac{r(x, z)}{\sqrt{t-\tau}} d \tau \leq C\left(\sup _{M \times[0, t)}|f|\right) r(x, z) t^{\frac{1}{2}} .
\end{aligned}
$$

Theorem 3.4. Let $h$ be a bounded function on $M$, and

$$
u(x, t)=\int_{M} H(x, y, t) h(y) d y
$$

for any $T>0$ and $0<t<T$, we have

(i)

$$
|\nabla u(x, t)| \leq C\left(\sup _{M}|h|\right) t^{-\frac{1}{2}}
$$


(ii) For $0<\alpha \leq 1$, and $[h]_{\alpha, M}=\sup _{x \neq y} \frac{|h(x)-h(y)|}{r^{\alpha}(x, y)}<\infty$,

$$
|u(x, t)-h(x)| \leq C[h]_{\alpha, M} t^{\frac{\alpha}{2}} .
$$

(iii) For $0<t_{1}<t_{2}<T$,

$$
\left|u\left(x, t_{2}\right)-u\left(x, t_{1}\right)\right| \leq C\left(\sup _{M}|h|\right)\left(\frac{t_{2}-t_{1}}{t_{1}}\right)^{\frac{1}{2}} .
$$

(iv) In addition, if $h$ is $C^{2}, \sup _{M}|\nabla h|<\infty$, and $\sup _{M}|\triangle h|<\infty$, then

$$
\sup _{M \times[0, t)}|\nabla u| \leq \sup _{M}|\nabla h|+C\left(\sup _{M}|\triangle h|\right) t^{\frac{1}{2}} .
$$

Proof. (i) By Lemma 3.1,

$$
\begin{aligned}
|u(x, t)-u(z, t)| & \leq \int_{M}|H(x, y, t)-H(z, y, t)||h(y)| d y \\
& \leq C\left(\sup _{M}|h|\right) r(x, z) t^{-\frac{1}{2}}
\end{aligned}
$$

(ii) By Lemma 3.2,

$$
\begin{aligned}
|u(x, t)-h(x)| & \leq \int_{M} H(x, y, t)|h(y)-h(x)| d y \\
& \leq[h]_{\alpha, M} \int_{M} H(x, y, t) r^{\alpha} d y \leq C[h]_{\alpha, M} t^{\frac{\alpha}{2}} .
\end{aligned}
$$

(iii) Since

$$
\begin{gathered}
u\left(x, t_{2}\right)=\int_{M} H\left(x, y, t_{2}-t_{1}\right) u\left(y, t_{1}\right) d y, \\
\left|u\left(y, t_{1}\right)-u\left(x, t_{1}\right)\right| \leq C\left(\sup _{M}|h|\right) r(x, y) t_{1}^{-\frac{1}{2}},
\end{gathered}
$$

therefore,

$$
\begin{aligned}
\left|u\left(x, t_{2}\right)-u\left(x, t_{1}\right)\right| & \leq C^{\prime} \sup _{M}|h| \int_{M} H\left(x, y, t_{2}-t_{1}\right) r(x, y) t_{1}^{-\frac{1}{2}} d y \\
& \leq C\left(\sup _{M}|h|\right)\left(\frac{t_{2}-t_{1}}{t_{1}}\right)^{\frac{1}{2}} .
\end{aligned}
$$

(iv) By using that $M$ has polynomial volume growth and the estimate of $H(x, y, t)$, we can prove (see Appendix)

$$
u_{t}=\int_{M} H(x, y, t) \triangle h(y) d y
$$


and $u_{t}$ is continuous on $t$ for $t \geq 0$. Thus

$$
u(x, t)=h(x)+\int_{0}^{t} u_{\tau} d \tau=h(x)+\int_{0}^{t} \int_{M} H(x, y, \tau) \triangle h(y) d y d \tau,
$$

therefore

$$
\begin{aligned}
|u(x, t)-u(z, t)| & \leq|h(x)-h(z)|+\int_{0}^{t} \int_{M}|H(x, y, \tau)-H(z, y, \tau)||\triangle h| d y d \tau \\
& \leq|h(x)-h(z)|+C^{\prime} \sup _{M}|\triangle h| \int_{0}^{t} \frac{r(x, z)}{\sqrt{\tau}} d \tau \\
& \leq|h(x)-h(z)|+C\left(\sup _{M}|\triangle h|\right) r(x, z) t^{\frac{1}{2}}
\end{aligned}
$$

\section{Short time solutions.}

Let $M, N$ be two complete Riemannian manifolds with metrics $g_{i j} d x^{i} d x^{j}$, $h_{\alpha \beta} d u^{\alpha} d u^{\beta}$, respectively. Suppose $h: M \rightarrow N$ is a $C^{1}$ map so that $h(M)$ is bounded in $N$, then there exists an open neighborhood $N^{\prime}$ of $h(M)$ with compact closure so that $N^{\prime}$ can be embedded into $R^{q}$ isometrically for some $q$. If necessary, by choosing a smaller neighborhood, we may assume that there exists a bounded tubular neighborhood $\tilde{N}$ of $N^{\prime}$ in $R^{q}$.

Let $\Pi: \tilde{N} \rightarrow N^{\prime}$ be the nearest point projection denoted by $\Pi=$ $\left(\Pi^{1}, \ldots, \Pi^{q}\right)=\left(\Pi^{A}\right)_{1 \leq A \leq q}$. By choosing an even smaller $N^{\prime}$, we may assume that $\Pi$ can be extended smoothly to the whole $R^{q}$ so that each $\Pi^{A}$ is compactly supported. Hence

$$
\Pi^{A}, \quad \Pi_{B}^{A}=\frac{\partial \Pi^{A}}{\partial z^{B}}, \quad \Pi_{B C}^{A}=\frac{\partial^{2} \Pi^{A}}{\partial z^{B} \partial z^{C}}, \ldots,
$$

are bounded, where $z=\left(z^{A}\right)$ are the standard coordinates of $R^{q}$.

Consider:

$$
\begin{gathered}
\left(\triangle-\frac{\partial}{\partial t}\right) u^{A}=\Pi_{B C}^{A}(u) \frac{\partial u^{B}}{\partial x^{i}} \frac{\partial u^{C}}{\partial x^{j}} g^{i j}=\Pi_{B C}^{A}(u) \nabla u^{B} \nabla u^{C}, \\
u(x, 0)=h(x) .
\end{gathered}
$$

Lemma 4.1. Suppose $u(N)$ lies in $N^{\prime}$, then $u$ satisfies the heat flow for harmonic maps from $M \times[0, \infty) \rightarrow N$ if and only if $u$ satisfies (4.1) and (4.2).

Proof. See [E-S, p. 140]. 
Lemma 4.2. Suppose that $M$ satisfies (a), $u$ is a solution of (4.1) and (4.2) which is continuous on $M \times[0, T)$ with $u(x, 0) \in N^{\prime}$ for all $x \in M$. If $u(x, t) \in \tilde{N}$ on $M \times[0, T)$, then $u(x, t) \in N^{\prime}$ for all $(x, t) \in M \times[0, T)$.

Proof. Since $M$ has polynomial volume growth, this lemma follows from [L-T, Lemma 3.2].

In what follows, we will prove the short time existence for the initial value problem of (4.1) and (4.2).

Theorem 4.3. Let $M$ be a complete noncompact Riemannian manifold without boundary and satisfy (a) and (b), $N$ be an arbitrary complete manifold. Given $h \in C^{2}(M, N)$ so that $h(M)$ is bounded in $N$, $\sup _{M} e(h)<\infty$ and $\sup _{M}|\tau(h)|<\infty$. Then there exists $T_{0}>0$ such that (4.1) and (4.2) has a unique solution on $M \times\left[0, T_{0}\right)$.

Proof. Set

$$
\Lambda=\sup _{R^{q}, A, B, C, D}\left(\left|\Pi_{B C}^{A}\right|,\left|\Pi_{B C D}^{A}\right|\right) .
$$

The hypotheses on $h$ imply that for $1 \leq A \leq q$,

$$
\sup _{M}\left|h^{A}\right|<\infty, \quad \sup _{M}\left|\nabla h^{A}\right|<\infty, \quad \sup _{M}\left|\triangle h^{A}\right|<\infty .
$$

For $\nu=-1,0,1,2, \ldots$, define

$$
u^{\nu}: M \times[0,1) \rightarrow R^{q}
$$

as follows:

$$
\begin{aligned}
u^{-1, A}(x, t) & =0 \\
u^{0, A}(x, t) & =\int_{M} H(x, y, t) h^{A}(y) d y
\end{aligned}
$$

for $\nu \geq 1$,

$$
u^{\nu, A}(x, t)=-\int_{0}^{t} \int_{M} H(x, y, t-\tau) F^{\nu-1, A}(y, \tau) d y d \tau+u^{0, A}(x, t)
$$

Where, for $\nu \geq-1, A=1, \ldots, q$,

$$
F^{\nu, A}=\Pi_{B C}^{A}(u) \nabla u^{\nu, B} \nabla u^{\nu, C} .
$$

Obviously, $F^{-1}=0, u^{-1}$ and $u^{0}$ are well-defined and smooth on $M \times(0,1)$. 
For $0<t<1$, let

$$
p_{\nu}(t)=\sup _{M \times(0, t)}\left(\sum_{A}\left|\nabla u^{\nu, A}\right|^{2}\right)^{\frac{1}{2}} .
$$

Clearly, $p_{\nu}(t)$ is nondecreasing in $t$, and

$$
\sup _{M \times(0, t)}\left|F^{\nu, A}\right| \leq C_{1}(m, q, \Lambda) p_{\nu}^{2}(t) .
$$

In order to prove that $u^{\nu}$ are well-defined, it suffices to show that $p_{\nu}(t)<$ $\infty$ for all $\nu$ and for all $0<t<1$.

When $\nu=0$, by Theorem 3.4(iv),

$$
p_{0}(t) \leq C_{2}(q)\left(\sup _{M, A}\left|\nabla h^{A}\right|+C \sup _{M, A}\left|\triangle h^{A}\right| t^{\frac{1}{2}}\right) .
$$

Hence $u^{1}$ is well-defined and is smooth on $M \times(0,1)$. Suppose $u^{\nu}$ is defined, $p_{\nu-1}(t)<\infty$ for $0<t<1$, and $u^{\nu}$ is smooth on $M \times(0,1)$. Theorem 3.3(ii) implies that for $0<t<1$,

$$
\begin{aligned}
\sup _{M \times(0, t)}\left|\nabla u^{\nu, A}\right| & \leq C_{3} t^{\frac{1}{2}} \sup _{M \times(0, t)}\left|F^{\nu-1, A}\right|+\sup _{M \times(0, t)}\left|\nabla u^{0, A}\right| \\
& \leq C_{4} t^{\frac{1}{2}} p_{\nu-1}^{2}(t)+\sup _{M \times(0, t)}\left|\nabla u^{0, A}\right| .
\end{aligned}
$$

Hence

$$
p_{\nu}(t) \leq C_{5} t^{\frac{1}{2}} p_{\nu-1}^{2}(t)+p_{0}(t)
$$

By induction hypothesis, we conclude that $u^{\nu+1}$ is well-defined and is smooth on $M \times(0, t)$.

Now choose $0<T_{1}<1$ such that

$$
C_{2} C_{5} T_{1}^{\frac{1}{2}}\left(\sup _{M, A}\left|\nabla h^{A}\right|+C \sup _{M, A}\left|\triangle h^{A}\right|\right) \leq \frac{1}{4},
$$

then

$$
C_{5} T_{1}^{\frac{1}{2}} p_{0}(t) \leq \frac{1}{4}
$$

If

$$
C_{5} T_{1}^{\frac{1}{2}} p_{\nu-1}(t) \leq \frac{1}{2}
$$


on $\left(0, T_{1}\right)$, (4.9) implies that for $0<t<T_{1}$,

$$
C_{5} T_{1}^{\frac{1}{2}} p_{\nu}(t) \leq\left(C_{5} T_{1}^{\frac{1}{2}} p_{\nu-1}(t)\right)^{2}+C_{5} T_{1}^{\frac{1}{2}} p_{0}(t) \leq \frac{1}{2} .
$$

Hence for all $\nu \geq 1$, on $\left(0, T_{1}\right)$,

$$
C_{5} T_{1}^{\frac{1}{2}} p_{\nu}(t) \leq \frac{1}{2}
$$

Thus $u^{\nu}$ are uniformly bounded on $M \times\left(0, T_{1}\right)$. Therefore the following function $X_{\nu}$ and $\bar{X}_{\nu}$ are well-defined,

$$
X_{\nu}(t)=\sup _{M} \sum_{A}\left|u^{\nu, A}-u^{\nu-1, A}\right|+\sup _{M}\left(\sum_{A}\left|\nabla u^{\nu, A}-\nabla u^{\nu-1, A}\right|^{2}\right)^{\frac{1}{2}},
$$

and, for $0<t<T_{1}$,

$$
\bar{X}_{\nu}(t)=\sup _{0<\tau<t} X_{\nu}(\tau)
$$

Now

$$
\begin{aligned}
F^{\nu, A}-F^{\nu-1, A}= & \Pi_{B C}^{A}\left(u^{\nu}\right) \nabla u^{\nu, B} \nabla u^{\nu, C}-\Pi_{B C}^{A}\left(u^{\nu-1}\right) \nabla u^{\nu-1, B} \nabla u^{\nu-1, C} \\
= & \left(\Pi_{B C}^{A}\left(u^{\nu}\right)-\Pi_{B C}^{A}\left(u^{\nu-1}\right)\right) \nabla u^{\nu, B} \nabla u^{\nu, C} \\
& +\Pi_{B C}^{A}\left(u^{\nu-1}\right)\left(\nabla u^{\nu, B}-\nabla u^{\nu-1, B}\right) \nabla u^{\nu, C} \\
& +\Pi_{B C}^{A}\left(u^{\nu-1}\right) \nabla u^{\nu-1, B}\left(\nabla u^{\nu-1, C}-\nabla u^{\nu-1, C}\right) .
\end{aligned}
$$

Applying the mean-value theorem to

$$
\Pi_{B C}^{A}\left(u^{\nu}\right)-\Pi_{B C}^{A}\left(u^{\nu-1}\right)
$$

we have

$$
\sup _{M \times(0, t)}\left|F^{\nu, A}-F^{\nu-1, A}\right| \leq C_{6} \bar{X}_{\nu}(t)\left(p_{\nu}^{2}(t)+p_{\nu}(t)+p_{\nu-1}(t)\right) .
$$

Inequality (4.12) asserts that for $0<t<T_{1}<1$,

$$
\sup _{M \times(0, t)}\left|F^{\nu, A}-F^{\nu-1, A}\right| \leq C_{7} \bar{X}_{\nu}(t) .
$$

Since

$$
u^{\nu+1, A}(x, t)-u^{\nu, A}(x, t)=-\int_{0}^{t} \int_{M} H(x, y, t-\tau)\left(F^{\nu, A}-F^{\nu-1, A}\right) d y d \tau
$$


by Theorem 3.3, we have

$$
\left|u^{\nu+1, A}(x, t)-u^{\nu, A}(x, t)\right| \leq t \sup _{M \times(0, t)}\left|F^{\nu, A}-F^{\nu-1, A}\right| \leq C_{7} t \bar{X}_{\nu}(t),
$$

and

$$
\begin{aligned}
& \left|\nabla u^{\nu+1, A}(x, t)-\nabla u^{\nu, A}(x, t)\right| \\
& \quad \leq C_{8} t^{\frac{1}{2}} \sup _{M \times(0, t)}\left|F^{\nu, A}-F^{\nu-1, A}\right| \leq C_{9} t^{\frac{1}{2}} \bar{X}_{\nu}(t) .
\end{aligned}
$$

Thus

$$
\bar{X}_{\nu+1}(t) \leq C_{10} t^{\frac{1}{2}} \bar{X}_{\nu}(t)
$$

Choose $0<T_{0}<1$ such that $C_{10} T_{0}^{\frac{1}{2}}<1$. If $0<t<T_{0}$, by Theorem 3.4(iii), we conclude that

$$
\begin{aligned}
\bar{X}_{\nu+1}(t) & \leq\left(C_{10} t^{\frac{1}{2}}\right)^{\nu} \bar{X}_{0}(t) \\
& \leq C_{11}\left(C_{10} T_{0}^{\frac{1}{2}}\right)^{\nu}\left(\sup _{M, A}\left|h^{A}\right|+\sup _{M, A}\left|\nabla h^{A}\right|+C \sup _{M, A}\left|\triangle h^{A}\right|\right) .
\end{aligned}
$$

Hence, $\sum_{\nu=1}^{\infty} X_{\nu}(t)$ converges uniformly on $\left(0, T_{0}\right)$. Thus $u^{\nu, A}$ and $\nabla u^{\nu, A}$ converges uniformly on $M \times\left(0, T_{0}\right)$. Let, for $A=1,2, \ldots, q$,

$$
u^{A}=\lim _{\nu \rightarrow \infty} u^{\nu, A} \text {. }
$$

Then $\nabla u^{A}$ exists and $\nabla u^{\nu, A} \rightarrow \nabla u^{A}$ uniformly on $M \times\left(0, T_{0}\right)$. Thus for all $A$,

$$
F^{\nu, A} \rightarrow F^{A}=\Pi_{B C}^{A}(u) \nabla u^{B} \nabla u^{C}
$$

uniformly on $M \times\left(0, T_{0}\right)$. Hence on $M \times\left(0, T_{0}\right)$, we have

$$
\begin{aligned}
u^{A}(x, t)= & -\int_{0}^{t} \int_{M} H(x, y, t-\tau) F^{A}(y, \tau) d y d \tau \\
& +\int_{M} H(x, y, t) h^{A}(y) d y .
\end{aligned}
$$

Note that each $u^{\nu}$ is smooth on $M \times\left(0, T_{0}\right)$ and satisfies, for $A=1, \ldots, q$,

$$
\left(\triangle-\frac{\partial}{\partial t}\right) u^{\nu, A}=F^{\nu-1, A} .
$$

By (4.5), (4.7) and (4.12), it is easy to see that $F^{\nu}$ and $u^{\nu}$ are uniformly bounded on $M \times\left(0, T_{0}\right)$. By [L-S-U, p. 211 Theorem 11.1], for any compact 
subset $\Omega \in M$ and $0<t_{1}<t_{2}<T_{0}$, there exists $C_{12}>0$ and $0<\mu<1$ independent of $\nu$ and $A$, such that

$$
\left|\nabla u^{\nu, A}(x, t)-\nabla u^{\nu, A}\left(x^{\prime}, t^{\prime}\right)\right| \leq C_{12}\left(r\left(x, x^{\prime}\right)^{\mu}+\left|t-t^{\prime}\right|^{\frac{\mu}{2}}\right),
$$

for all $x, x^{\prime} \in \Omega$ and for all $t_{1}<t, t^{\prime}<t_{2}$.

Letting $\nu \rightarrow \infty$, we have

$$
\left|\nabla u^{A}(x, t)-\nabla u^{A}\left(x^{\prime}, t^{\prime}\right)\right| \leq C_{12}\left(r\left(x, x^{\prime}\right)^{\mu}+\left|t-t^{\prime}\right|^{\frac{\mu}{2}}\right) .
$$

Hence by (4.20) one can conclude that $u(x, t)$ satisfies (4.1) in $M \times\left(0, T_{0}\right)$, and

$$
\lim _{t \rightarrow 0} u^{A}(x, t)=h^{A}(x) .
$$

Obviously, $T_{0}$ depends only on the geometries of $M, N$ and a neighborhood of $h(M)$ and the bounds of $e(h)$ and $\tau(h)$. Furthermore, the energy density and the image of the solution $u$ is bounded on $M \times\left[0, T_{0}\right)$ by a constant depending only on the known quantities mentioned above. $\lim _{t \rightarrow 0} u(x, t)=h(x)$ is uniformly on $M$ and $\lim _{t \rightarrow 0} e(u)(x, t)=e(h)$ is uniformly on compact subsets.

Uniqueness follows from [L-T, Theorem 3.5] since $M$ has polynomial volume growth.

\section{Long time solutions, harmonic maps.}

In this section, we will consider the long time solutions of the heat flow and the convergence to harmonic maps via mean-value inequalities.

Theorem 5.1. Suppose, under the conditions of Theorem 4.3, Riem $^{N} \leq k$ $(k \geq 0)$. Let $T^{*}$ be the supremum of these $T$ such that (1.1) has a unique solution $u(x, t)$ on $M \times[0, T)$ and $\sup _{M \times[0, T)} e(u)<\infty$. Then either $T^{*}=\infty$ or $T^{*}<\infty$ and $\lim _{T \rightarrow T^{*}} \sup _{M \times[0, T)} e(u)=\infty$.

Proof. The proof of the Theorem 4.3 implies that $T^{*}>0$. Suppose $T^{*}<\infty$ and

$$
\sup _{M \times\left[0, T^{*}\right)} e(u) \leq s<\infty .
$$

For the proof of the theorem, we need only show that the solution of (1.1) can be extended from $T^{*}$. By $[\mathbf{H}]$, we have, on $M \times\left[0, T^{*}\right)$,

$$
\left(\triangle-\frac{\partial}{\partial t}\right)\left|u_{t}\right|^{2} \geq-2 k s\left|u_{t}\right|^{2} \text {. }
$$


Hence $g(x, t)=\exp (-2 k s t)\left|u_{t}\right|^{2}$ is a positive subsolution of the heat equation for functions on $M$. As in [Lo-T], there exists $C_{1}>0$ depending only on dimension of $M$ such that for $t_{2}>t_{1} \geq 0$

$$
2 \int_{t_{1}}^{t_{2}} \int_{B_{p}(R)}\left|u_{t}\right|^{2} \leq E_{p}\left(t_{1}, 2 R\right)+\frac{C_{1}}{R^{2}} \int_{t_{1}}^{t_{2}} E_{p}(\tau, 2 R)
$$

where $E_{p}(\tau, R)=\int_{B_{p}(R)} e(u)(\cdot, \tau)$.

Let $\delta=\frac{1}{2}$ in Theorem 2.2. If $T^{*}<\infty$, by (2.7), for $T<T^{*}$,

$$
\sup _{B_{p}\left(\frac{R}{2}\right) \times[0, T]} g \leq \frac{2^{2+\beta} C}{R^{2} V_{p}(R)} \int_{0}^{T} \int_{B_{p}(R)} g+\sup _{M}|g(\cdot, 0)| .
$$

Since $E_{p}(\tau, R) \leq s V_{p}(R)$ for $\tau \geq 0$, by (5.1),

$$
\begin{aligned}
\sup _{B_{p}\left(\frac{R}{2}\right) \times[0, T]}\left|u_{t}\right|^{2} & \leq \frac{2^{2+\beta} C \exp (2 k s T)}{R^{2} V_{p}(R)} \int_{0}^{T} \int_{B_{p}(R)}\left|u_{t}\right|^{2}+\sup _{M}|\tau(h)|^{2} \\
& \leq 2^{1+\beta} C s \exp \left(2 k s T^{*}\right) \frac{V_{p}(2 R)}{V_{p}(R)}\left(\frac{1}{R^{2}}+\frac{C_{1} T^{*}}{R^{4}}\right)+\sup _{M}|\tau(h)|^{2}, \\
& \leq C^{\prime}\left(C, \beta, k, s, A, T^{*}\right)\left(\frac{1}{R^{2}}+\frac{C_{1} T^{*}}{R^{4}}\right)+\sup _{M}|\tau(h)|^{2},
\end{aligned}
$$

therefore

$$
\sup _{M \times[0, T]}\left|u_{t}\right|=\lim _{R \rightarrow \infty} \sup _{B_{p}\left(\frac{R}{2}\right) \times[0, T]}\left|u_{t}\right| \leq \sup _{M}|\tau(h)|<\infty .
$$

Thus

$$
\lim _{T \rightarrow T^{*}}|\tau(u)|(\cdot, T)=\lim _{T \rightarrow T^{*}}\left|u_{t}\right| \leq \sup _{M}|\tau(h)|<\infty .
$$

This estimate also implies that $u\left(M \times\left[0, T^{*}\right)\right)$ is bounded in $N$, and by the assumption

$$
\lim _{T \rightarrow T^{*}} e(u)(\cdot, T)<\infty,
$$

thus we can extend $u$ as a unique solution of (1.1) because of Theorem 4.3 and [L-T, Theorem 3.5]. This contradicts the definition of $T^{*}$ and the theorem follows.

Theorem 5.2. Let $M$ satisfy (a) and (b), Riem ${ }^{N} \leq 0$. Suppose (1.1) has a long-time solution $u(x, t)$ on $M \times[0, \infty)$. Moreover, suppose

$$
s(t)=\sup _{M \times[0, t)} e(u)(x, t)=O(\log t) .
$$


If there exists a point $p \in M$ and a sequence $t_{\nu} \rightarrow \infty$ such that sequence $u\left(p, t_{\nu}\right)$ converges in $N$, then $u\left(\cdot, t_{\nu}\right)$ converges uniformly on compact subsets of $M$ to a harmonic map $u_{\infty}$ by passing to a subsequence.

Proof. Choose $R^{2}=4 T, \tau=T$ in (2.5), then $\sigma=\frac{R^{2}}{4}$. Since $\left|u_{t}\right|^{2}$ satisfies (2.4) on $M \times[0, \infty)$, by $(2.5)$ and $(5.1)$,

$$
\begin{aligned}
\sup _{B_{p}(\sqrt{T})}\left|u_{t}\right|^{2}(\cdot, T) & \leq \frac{2^{2+\beta} C}{R^{2} V_{p}(R)} \int_{0}^{T} \int_{B_{p}(R)}\left|u_{t}\right|^{2} \\
& \leq 2^{1+\beta} C\left(1+\frac{C_{1}}{4}\right) \frac{V_{p}(2 R) s(T)}{V_{p}(R) R^{2}} \\
& \leq C^{\prime}\left(C, C_{1}, \beta, A\right) \frac{s(T)}{T},
\end{aligned}
$$

therefore, let $T \rightarrow \infty$, if $s(T)=O(\log T)$, we have, on $M$,

$$
\tau\left(u_{\infty}\right)=\lim _{T \rightarrow \infty} \sup _{M}\left|u_{t}\right|(\cdot, T)=0 .
$$

Hence, the theorem follows.

\section{Appendix.}

In this appendix, we will use the argument of $[\mathbf{L i 1}],[\mathbf{L i 2}]$ to prove the following proposition:

Proposition. Let $M$ be complete noncompact manifold which satisfies (a) and (b), $f$ is a $C^{2}$ function on $M$ and

$$
\sup _{M}|f|<\infty, \quad \sup _{M}|\nabla f|<\infty, \quad \sup _{M}|\triangle f|<\infty .
$$

Suppose

$$
u(x, t)=\int_{M} H(x, y, t) f(y) d y
$$

then, for $t>0$,

$$
u_{t}(x, t)=\int_{M} H(x, y, t) \triangle f(y) d y
$$

Proof. By the Green's identity,

$$
\left|\int_{B_{x}(R)} \triangle_{y} H(x, y, t) f(y) d y-\int_{B_{x}(R)} H(x, y, t) \triangle f(y) d y\right|
$$




$$
\begin{aligned}
& =\left|\int_{\partial B_{x}(R)} \frac{\partial H}{\partial \nu_{y}}(x, y, t) f(y) d y-\int_{\partial B_{x}(R)} H(x, y, t) \frac{\partial f}{\partial \nu_{y}}(y) d y\right| \\
& \leq \int_{\partial B_{x}(R)}\left|\nabla_{y} H\right|(x, y, t) f(y) d y+\int_{\partial B_{x}(R)} H(x, y, t)|\nabla f|(y) d y
\end{aligned}
$$

$$
\leq \sup _{M}|f| \int_{\partial B_{x}(R)}\left|\nabla_{y} H\right|(x, y, t) d y+\sup _{M}|\nabla f| \int_{\partial B_{x}(R)} H(x, y, t)(y) d y .
$$

For the heat kernel $H(x, y, t)$ of any complete manifold $M$, we have (see [C-L-Y], (4.18))

$$
\begin{aligned}
& \int_{M \backslash B_{x}\left(\frac{3}{4} R\right)}|\nabla H|^{2} \\
& \leq\left(\int_{M \backslash B_{x}\left(\frac{1}{2} R\right)} H^{2}\right)^{\frac{1}{2}}\left[\frac{64}{R^{2}}\left(\int_{M} H^{2}\right)^{\frac{1}{2}}+2\left(\int_{M}(\triangle H)^{2}\right)^{\frac{1}{2}}\right] .
\end{aligned}
$$

In terms of the argument of [C-L-Y, p. 1052-1055] and the upper estimate of the heat kernel (Theorem 2.1(iii)), we can prove

$$
\begin{gathered}
\int_{M} H^{2}(x, y, t) d y=H(x, x, 2 t) \leq \frac{1}{C_{2} V_{x}(\sqrt{2 t})}, \\
\int_{M}(\triangle H)^{2}(x, y, t) d y \leq \frac{2}{t^{2}} H(x, x, t) \leq \frac{4}{C_{2} t^{2} V_{x}(\sqrt{t})},
\end{gathered}
$$

and

$$
\int_{M \backslash B_{x}\left(\frac{1}{2} R\right)} H^{2}(x, y, t) d y \leq \frac{1}{C_{2} V_{x}(\sqrt{t})} \exp \left(-\frac{C_{4} R^{2}}{4 t}\right) .
$$

Substituing (6.3), (6.4) and (6.5) into (6.2), since $M$ has polynomial volume growth (Theorem 2.1(i)), we can find constants $C>0, \delta>0$ such that for all $t>0$,

$$
\int_{B_{x}(R) \backslash B_{x}\left(\frac{3}{4} R\right)}|\nabla H|^{2}(x, y, t) d y \leq C^{2}\left(\frac{1}{R^{2}}+\frac{1}{t}\right) t^{-2 \delta} \exp \left(-\frac{C_{4} R^{2}}{8 t}\right) .
$$

Then, the Holder's inequality implies

$$
\begin{aligned}
\int_{\partial B_{x}(R)}|\nabla H|(x, y, t) d y & \leq \int_{B_{x}(R) \backslash B_{x}\left(\frac{3}{4} R\right)}|\nabla H|(x, y, t) d y \\
& \leq C\left(\frac{1}{R}+\frac{1}{\sqrt{t}}\right) t^{-\delta} V_{x}^{\frac{1}{2}}(R) \exp \left(-\frac{C_{4} R^{2}}{16 t}\right) .
\end{aligned}
$$


On the other hand, the upper estimate of the heat kernel implies

$$
\int_{\partial B_{x}(R)} H(x, y, t) d y \leq \frac{V_{x}(R)}{C_{2} V_{x}(\sqrt{t})} \exp \left(-\frac{C_{4} R^{2}}{t}\right) .
$$

Substituting (6.6), (6.7) into (6.1), thus, let $R \rightarrow \infty$, we have

$$
\left|\int_{M} \triangle_{y} H(x, y, t) f(y) d y-\int_{M} H(x, y, t) \triangle f(y) d y\right|=0 .
$$

Since

$$
\begin{aligned}
\left|\int_{M} H_{t}(x, y, t) f(y) d y\right| & =\left|\int_{M} \triangle_{y} H(x, y, t) f(y) d y\right| \\
& =\left|\int_{M} H(x, y, t) \triangle f(y) d y\right| \leq \sup _{M}|\triangle f|<\infty,
\end{aligned}
$$

therefore

$u_{t}=\int_{M} H_{t}(x, y, t) h(y) d y=\int_{M} \triangle_{y} H(x, y, t) h(y) d y=\int_{M} H(x, y, t) \triangle h(y) d y$.

\section{References}

[C] S.Y. Cheng, Liouville theorem for harmonic maps, Proc. Symp. Pure Math., 36 (1980), 147-151.

[C-L-Y] S.Y. Cheng, P. Li and S.T. Yau, On the upper estimate of the heat kernel of a Riemannian manifold, Amer. J. Math., 103 (1981), 1021-1063.

[E-S] J. Eells and J. Sampson, Harmonic mappings of Riemannian manifolds, Amer. J. Math., 86 (1964), 109-160.

[G] A.A. Grigor'yan, The heat equation on noncompact Riemannian manifolds, (in Russian), Matem. Sbornik, 182(1) (1991), 55-87; Engl. Transl. in Math. USSR Sb., 72(1) (1992), 47-77.

[H] R. Hamilton, Harmonic mapping between Riemannian manifolds, Lecture Notes, 471 (1975), Springer.

[L-S-U] O.A. Ladyzenskaja, V.A. Solonnikov and N.N. Ural'ceva, Linear and quasilinear equations of parabolic type, Amer. Math. Soc. Transl. Math. Monographs, 23, Providence, 1968.

[L] J. Li, The heat flows and harmonic maps of complete noncompact Riemannian manifolds, Math. Z., 212 (1993), 161-173.

[Li1] P. Li, Uniqueness of $L^{1}$ solutions for the Laplace equation and the heat equation on Riemannian manifolds, J. Diff. Geom., 20 (1984), 447-457.

[Li2] L Large time behavior of the heat equation on complete manifolds with nonnegative Ricci curvature, Ann. Math., 124 (1986), 1-21. 
[L-T] P. Li and L-F. Tam, The heat equation and harmonic maps of complete manifolds, Invent. Math., 105 (1991), 1-46.

[Lo-T] G. Liao and L-F. Tam, On the heat equation for harmonic maps from non-compact manifolds, Pacific J. Math., 153(1) (1992), 129-145.

[S-Y1] R. Schoen and S-T. Yau, Harmonic maps and the topology of stable hypersurfaces and manifolds of nonnegative Ricci curvature, Comm. Math. Helv., 39 (1976), 333-341.

[S-Y2] C Compact group actions and the topology of manifolds with nonpositive curvature, Topology, 18 (1979), 361-380.

[T1] L-F. Tam, Liouville property for harmonic maps on a class of manifolds, preprint.

[T2] , preprint.

[W] J. Wang, The heat flow and harmonic maps between complete manifolds, thesis, 1994, University of California, Irvine.

Received March 18, 1996.

Morningside Center of Mathematics and Institute of Mathematics Chinese ACADEmy of Sciences

BeIJING 100080, P.R. China

E-mail address: xzhang@math03.math.ac.cn

Note: The PAPER Version DeRIVEd (6.8) By SUbSTituting (6.7) AND (6.8) (RATHeR THAN (6.6) AND (6.7)) INTO (6.1). 